\title{
Characterizations of Kumaraswamy-geometric distribution
}

\author{
G. G. Hamedani
}

\section{Correspondence:}

g.hamedani@mu.edu

Department of Mathematics,

Statistics and Computer Science,

Marquette University, Milwaukee,

WI 53201-1881, USA

\section{Abstract \\ Certain characterizations of Kumaraswamy-geometric distribution introduced by Akinsete et al. (JSDA 1:1-21, 2014) are presented.}

\section{Introduction}

The problem of characterizing a distribution is an important problem in applied sciences, where an investigator is vitally interested to know if their model follows the right distribution. To this end the investigator relies on conditions under which their model would follow specifically chosen distribution. Akinsete et al. (2014) introduced a distribution called Kumaraswamy-geometric distribution (KGD) and studied various properties of the distribution. In this very short note, we present two characterizations of KGD based on: (i) Conditional expectation of certain function of the random variable and (ii) the reverse hazard rate function.

The cumulative distribution function (cdf) of KGD and its corresponding probability mass function (pmf) are given, respectively, by

$$
G(x)=1-\left[1-\left(1-q^{x+1}\right)^{\alpha}\right]^{\beta}, x=0,1,2, \ldots
$$

and

$$
g(x)=\left[1-\left(1-q^{x}\right)^{\alpha}\right]^{\beta}-\left[1-\left(1-q^{x+1}\right)^{\alpha}\right]^{\beta}, x=0,1,2, \ldots
$$

where $q=1-p$ and $p$ is the parameter of the geometric distribution.

We rewrite $g(x)$ as

$$
g(x)=\exp \left\{\beta \log \left[\left[1-\left(1-q^{x}\right)^{\alpha}\right]\right]\right\}-\exp \left\{\beta \log \left[\left[1-\left(1-q^{x+1}\right)^{\alpha}\right]\right]\right\} .
$$

The hazard rate function of KGD is given by

$$
h_{g}(x)=\exp \left\{\beta \log \left[\left[1-\left(1-q^{x}\right)^{\alpha}\right]\right]-\beta \log \left[\left[1-\left(1-q^{x+1}\right)^{\alpha}\right]\right]\right\}-1,
$$

and its reverse hazard rate function for $\beta=1$, by

$$
r_{g}(x)=1-\exp \left\{\alpha \log \left[\left(1-q^{x}\right)\right]-\alpha \log \left[\left(1-q^{x+1}\right)\right]\right\} .
$$

\section{Characterization results}

In what follows we use $\mathbb{N}^{*}$ for $\{0\} \cup \mathbb{N}$ and present our characterizations via two subsections.

(c) 2016 Hamedani. Open Access This article is distributed under the terms of the Creative Commons Attribution 4.0 International License (http://creativecommons.org/licenses/by/4.0/), which permits unrestricted use, distribution, and reproduction in any medium, provided you give appropriate credit to the original author(s) and the source, provide a link to the Creative Commons license, and indicate if changes were made. 


\subsection{Characterization of KGD in terms of the conditional expectation of certain function of the random variable}

Proposition 2.1.1. Let $X: \Omega \rightarrow \mathbb{N}^{*}$ be a random variable. The pmf of $X$ is (3) if and only if

$$
\begin{aligned}
& E\left\{\left[\exp \left\{\beta \log \left[\left[1-\left(1-q^{x}\right)^{\alpha}\right]\right]\right\}+\exp \left\{\beta \log \left[\left[1-\left(1-q^{x+1}\right)^{\alpha}\right]\right]\right\}\right] \mid X>k\right\} \\
= & \exp \left\{\beta \log \left[\left[1-\left(1-q^{k+1}\right)^{\alpha}\right]\right]\right\} .
\end{aligned}
$$

Proof. If $X$ has pmf (3), then the left-hand side of (6) will be

$$
\begin{aligned}
& (1-G(k))^{-1} \sum_{x=k+1}^{\infty}\left\{\exp \left\{2 \beta \log \left[\left[1-\left(1-q^{x}\right)^{\alpha}\right]\right]\right\}-\exp \left\{2 \beta \log \left[\left[1-\left(1-q^{x+1}\right)^{\alpha}\right]\right]\right\}\right\} \\
= & \left(\exp \left\{-\beta \log \left[\left[1-\left(1-q^{k+1}\right)^{\alpha}\right]\right]\right\}\right)\left(\exp \left\{2 \beta \log \left[\left[1-\left(1-q^{k+1}\right)^{\alpha}\right]\right]\right\}\right) \\
= & \exp \left\{\beta \log \left[\left[1-\left(1-q^{k+1}\right)^{\alpha}\right]\right]\right\} .
\end{aligned}
$$

Conversely, if (6) holds, then

$$
\begin{aligned}
& \sum_{x=k+1}^{\infty}\left\{\left[\exp \left\{\beta \log \left[\left[1-\left(1-q^{x}\right)^{\alpha}\right]\right]\right\}+\exp \left\{\beta \log \left[\left[1-\left(1-q^{x+1}\right)^{\alpha}\right]\right]\right\}\right] g(x)\right\} \\
= & (1-G(k)) \exp \left(\beta \log \left[\left[1-\left(1-q^{k+1}\right)^{\alpha}\right]\right]\right) \\
= & \{(1-G(k+1))+g(k+1)\} \exp \left(\beta \log \left[\left[1-\left(1-q^{k+1}\right)^{\alpha}\right]\right]\right)
\end{aligned}
$$

From (6), we also have

$$
\begin{aligned}
& \sum_{x=k+2}^{\infty}\left\{\left[\exp \left\{\beta \log \left[\left[1-\left(1-q^{x}\right)^{\alpha}\right]\right]\right\}+\exp \left\{\beta \log \left[\left[1-\left(1-q^{x+1}\right)^{\alpha}\right]\right]\right\}\right] g(x)\right\} \\
= & (1-G(k+1)) \exp \left(\beta \log \left[\left[1-\left(1-q^{k+2}\right)^{\alpha}\right]\right]\right) .
\end{aligned}
$$

Now, subtracting (8) from (7), yields

$$
\begin{aligned}
& \exp \left(\beta \log \left[\left[1-\left(1-q^{k+2}\right)^{\alpha}\right]\right]\right) g(k+1) \\
= & (1-G(k+1))\left\{\exp \left\{\beta \log \left[\left[1-\left(1-q^{k+1}\right)^{\alpha}\right]\right]\right\}-\exp \left\{\beta \log \left[\left[1-\left(1-q^{k+2}\right)^{\alpha}\right]\right]\right\}\right\} .
\end{aligned}
$$

From the above equality, we have

$$
\begin{aligned}
h_{g}(k+1)= & \frac{g(k+1)}{(1-G(k+1))}= \\
& \frac{\left\{\exp \left\{\beta \log \left[\left[1-\left(1-q^{k+1}\right)^{\alpha}\right]\right]\right\}-\exp \left\{\beta \log \left[\left[1-\left(1-q^{k+2}\right)^{\alpha}\right]\right]\right\}\right\}}{\exp \left(\beta \log \left[\left[1-\left(1-q^{k+2}\right)^{\alpha}\right]\right]\right)} \\
= & \exp \left\{\beta \log \left[\left[1-\left(1-q^{k+1}\right)^{\alpha}\right]\right]-\beta \log \left[\left[1-\left(1-q^{k+2}\right)^{\alpha}\right]\right]\right\}-1,
\end{aligned}
$$

which, in view of (4), implies that $X$ has mpf (3).

Remark 2.1.1. For $\beta=1$, KGD reduces to EEGD (Exponentiated Exponential Geometric Distribution) defined by Alzaatreh et al. (JSM 9:589-603, 2012). 


\subsection{Characterization of KGD based on reverse hazard function}

Proposition 2.2.1. Let $X: \Omega \rightarrow \mathbb{N}^{*}$ be a random variable. For $\beta=1$, the pmf of $X$ is

(2) if and only if its reverse hazard rate function satisfies the difference equation

$$
r_{g}(k+1)-r_{g}(k)=\left(\frac{1-q^{k}}{1-q^{k+1}}\right)^{\alpha}-\left(\frac{1-q^{k+1}}{1-q^{k+2}}\right)^{\alpha}, k \in \mathbb{N}^{*},
$$

with the initial condition $r_{g}(0)=1$.

Proof. If $X$ has pmf (2) for $\beta=1$, then clearly (9) holds. Now, if (9) holds, then for every $x \in \mathbb{N}$, we have

$$
\sum_{k=0}^{x-1}\left\{r_{g}(k+1)-r_{g}(k)\right\}=\sum_{k=0}^{x-1}\left\{\left(\frac{1-q^{k}}{1-q^{k+1}}\right)^{\alpha}-\left(\frac{1-q^{k+1}}{1-q^{k+2}}\right)^{\alpha}\right\},
$$

or

$$
r_{g}(x)-r_{g}(0)=-\left(\frac{1-q^{x}}{1-q^{x+1}}\right)^{\alpha},
$$

or

$$
r_{g}(x)=1-\left(\frac{1-q^{x}}{1-q^{x+1}}\right)^{\alpha}, x \in \mathbb{N}^{*}
$$

which, in view of the reverse hazard rate function (5), $X$ has pmf (2).

\subsection{Further observation}

Proposition 2.3.1. Let $X_{1}, X_{2}, \ldots, X_{n}$ be $n$ independent random variables with $X_{i} \sim$ $K G D\left(\alpha, \beta_{i}\right), i=1,2, \ldots, n$. Then $X_{\min }=\min \left\{X_{1}, X_{2}, \ldots, X_{n}\right\} \sim K G D\left(\alpha, \sum_{i=1}^{n} \beta_{i}\right)$.

Proof. It follows from

$$
\begin{aligned}
P\left(X_{\min }>x\right) & =\left[P\left(X_{i}>x\right)\right]^{n} \\
& =\Pi_{i=1}^{n}\left[1-\left(1-q^{x+1}\right)^{\alpha}\right]^{\beta_{i}} \\
& =\left[1-\left(1-q^{x+1}\right)^{\alpha}\right]^{\sum_{i=1}^{n} \beta_{i}} .
\end{aligned}
$$

\section{Acknowledgement}

The author is grateful to a referee who pointed out an error in Eq. (9) in the original version of this short note. The author thanks an Associate Editor for suggesting the addition of the important second reference.

Received: 1 December 2015 Accepted: 29 December 2015

Published online: 16 January 2016

References

Akinsete, A, Famoye, F, Lee, C: The Kumaraswamy-geometric distribution. JSDA. 1(17), 1-21 (2014)

Alzaatreh, A, Lee, C, Famoye, F: On discrete analogues of continuous distributions. Stat Methodol. 9, 589-603 (2012) 